\title{
Auto-Tuning of Aberrations Using High-Resolution STEM Images by Auto-Correlation Function
}

\author{
H. Sawada ${ }^{*}$, M. Watanabe ${ }^{* *}$, E. Okunishi ${ }^{*}$, and, Y. Kondo ${ }^{*}$ \\ *JEOL Ltd., 3-1-2 Musashino, Akishima, Tokyo, 196-8558, Japan \\ ** Dept. of Mater. Sci. and Eng., Lehigh University, Bethlehem. PA 18015.
}

Spherical aberration correctors in scanning transmission electron microscopes (STEMs) have been widely used for high-resolution structural and chemical analytical studies. A recent aberration corrector has the ability to compensate for residual aberrations up to fourth-order and a fifth-order spherical aberration as well as a third-order spherical aberration. Parasitic aberrations can be canceled out by adjusting the ray path using the optical elements in practical operations. To correct these aberrations by software, aberration measurements in the probe-forming lens system is essential. Recently, one of the authors has developed a measurement procedure of aberrations by auto-correlation function from a Ronchigram, called the Segmental Ronchigram Auto-correlation function Matrix (SRAM) method [1]. In this approach, the auto-correlation functions obtained from segmented areas of the Ronchigram are utilized to estimate a second-order differential of wave aberration. By collecting these auto-correlation functions from each angular area segmented in the Ronchigram, aberrations for the probe-forming system can be calculated. Several other methods to measure aberrations have also been reported by using a standard specimen of metallic particles [2] or Ronchigrams [3-7]. However, final fine adjustments of defocus and two-fold astigmatism are usually performed in a manual fashion during actual observation of atomic resolution images by experienced users. If aberrations are measurable from atomic-resolution STEM images of a real crystalline specimen directly, aberration auto-tuning could be applied instantaneously during structural image observation before recording final images. Therefore, the aberration measurement method, the Segmental Image Auto-correlation function Matrix (SIAM) method, has been developed, based on a series of auto-correlation functions from segmented areas of high-resolution STEM images from a crystalline specimen. In this paper, the principles and experimental results of the SIAM method will be reported.

Figure 1(b) shows an auto-correlation function pattern from the selected central part of Fig.1 (a). A high-angle annular dark-field (HAADF) STEM image can be assumed as a convolution of a probe shape function and a map of intensity of highly scattered electrons, which reveals atomic (or atomic column) arrangement of a specimen. In the SIAM method, the auto-correlation function from a HAADF-STEM image is supposed to be a probe shape function with residual aberrations, if it can be assumed that the intensity map of each atomic column is isotropic. An elongated ellipse in the auto-correlation function pattern shown in Fig. 1(b) indicates that the probe in the STEM image contains a two-fold astigmatism in some degree. The auto-correlation function is calculated from a small segmented area in the STEM image to reduce the periodicity from crystalline lattice. Then, the values of defocus and two-fold astigmatism are measured from the central peak in the auto-correlation pattern by fitting to ellipse shape (Fig.1 (b)). Two sets of defocused STEM images are required to determine the absolute values of these aberrations, which are converted from the known defocus values. Multiple auto-correlation patterns from an image are used for the calculation of the aberration values to average individual variation of specimen structure in each segmented area (Fig.1 (c)). Appropriate shift values of the focus and stigmators to compensate 
measured aberrations fed into the instrument automatically through a script in the Gatan DigitalMicrograph platform in this study. Figure 2 compares high-resolution HAADF-STEM images of $\mathrm{SrTiO}_{3}$ (a) before and (b) after applying the SIAM method, which were taken in a JEM-ARM200F instrument. Since two-fold astigmatism was intentionally introduced, lattice fringes were hardly visible as shown in Fig. 2(a). By applying the SIAM method using two sets of STEM images with defocuses of $\pm 10 \mathrm{~nm}$, the atomic-resolution lattice fringes now appear clearly without any manual adjustment. The SIAM auto fine-tuning procedure assists users to obtain an atomic-resolution image in aberration corrected STEM. This method can also be applicable to measurements of other higher-order aberrations, e.g. axial coma, introduced by tilt of the incident beam from the optical axis.

\section{Reference}

[1] H. Sawada et al, Ultramicrosc. 108 (2008) 1467.

[2] M. Haider et al, Ultramicroscopy 81 (2000) 163.

[3] J. M. Cowley, J. Electron Microscope Technique 3 (1986) 25.

[4] N. Dellby et al, J. Electron Microsc. 50 (2001) 177.

[5] Q. M. Ramasse and A. L. Bleloch, Ultramicrosc. 106 (2005) 37.

[6] T. Yamazaki et al, Ultramicrosc. 106 (2006) 153.

[7] A. R. Lupini et al, Ultramicrosc. 110 (2010) 891.

[8] The author (MW) wishes to acknowledge financial support from the NSF through grant DMR-0804528.
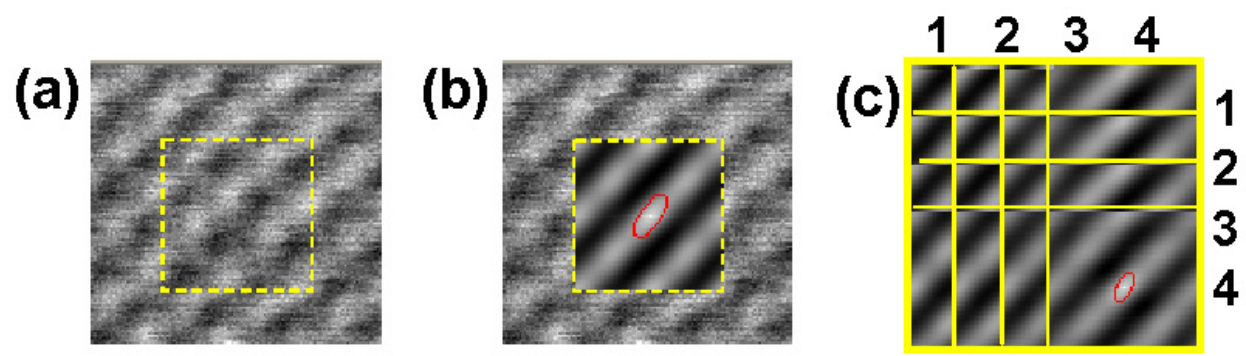

Fig. 1: (a) Defocused dark field STEM image of $\mathrm{SrTiO}_{3}$ with a size of 128 x 128 pixels. (b) The pattern of the auto-correlation function from 64 x 64 pixel in Fig. 1 (a), and ellipse fitting as shown by a red line. (c) $4 \times 4$ segmentations for auto-correlation patterns. Note that each pattern has overlapped region, since the area for auto-correlation is 64 x 64 in this figure.
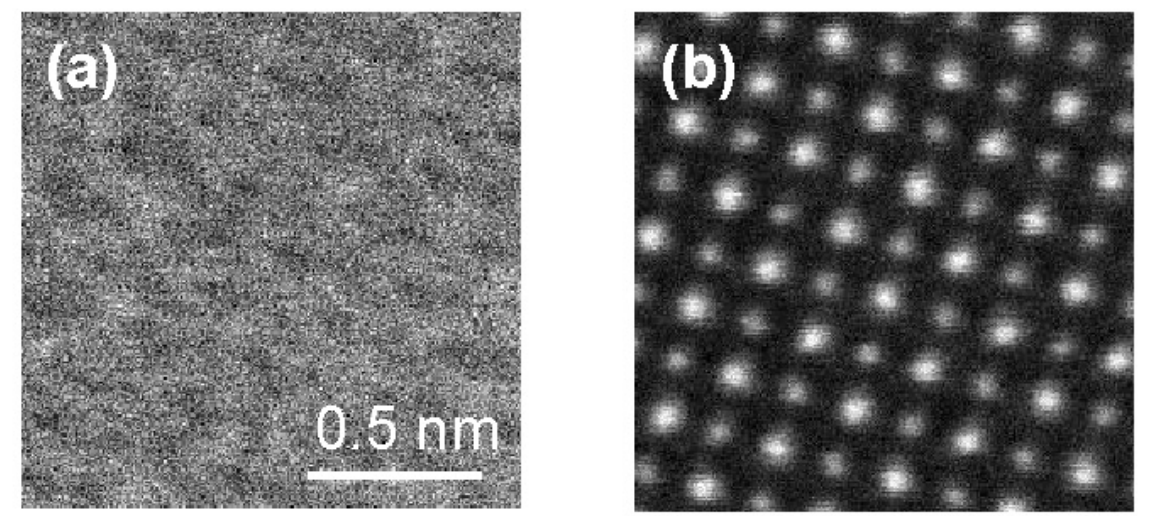

Fig. 2: High resolution images of $\mathrm{SrTiO}_{3}$ (a) before and (b) after five iterations of SIAM process. 